\title{
Performance Enhancement of the IMM Estimation by Smoothing*
}

\author{
Vesselin P. Jilkov \\ X. Rong Li \\ Lei Lu \\ Department of Electrical Engineering \\ University of New Orleans, New Orleans, LA 70148 \\ vjilkov@uno.edu, xli@uno.edu, 504-280-7416,-3950 (fax)
}

\begin{abstract}
This paper proposes and investigates an enhancement of the IMM estimation (referred to as smoothing-enhanced IMM or SE-IMM) at the expense of a reasonable increase in computation. It is based on improved reinitialization of the IMM filter by IMM smoothing. An approximate IMM smoothing (IMMS) algorithm is provided. To maintain a minimum overall computational load, the SE-IMM scheme is applied only in the transitional regimes of the IMM filter when the dominant model changes. A cumulative sums (CUSUM)-type statistical test is formulated and employed for the problem of model change detection/estimation. An investigation of the efficiency of the proposed smoothing-enhanced IMM estimation is performed by simulation of a maneuvering target tracking scenario.
\end{abstract}

Keywords: Multiple Model, IMM, Smoothing, Target Tracking, CUSUM

\section{Introduction}

While being one of the most cost-effective algorithms for tracking maneuvering targets, the IMM filter $[3,15,1]$ may still have quite large peek errors during transition between dominant models. Thus in cases of tracking intensively maneuvering targets the overall estimation error may be considerable.

This study proposes and investigates an enhancement of the IMM estimation (referred to as smoothing-enhanced IMM or SE-IMM), especially during transitional regimes, at the expense of a reasonable increase in computation. It is based on the idea that the IMM recursive cycle can be improved by N-step-back MM smoothing. For this purpose an approximate multiple-step-back IMM smoothing (IMMS) algorithm is provided. Generally, the SE-IMM operates as follows: at each time step after the IMM cycle is completed, an N-step-back MM smoother (e.g., IMMS) is used to obtain the smoothed model-conditional estimates

\footnotetext{
${ }^{*}$ Research Supported in part by ONR grant N00014-00-1-0677, NSF grant ECS-9734285, and NASA/LEQSF grant (2001-4)-01.
}

at some previous time step. Then the IMM filter is reinitialized with these smoothed estimates starting from that reference moment. The underlying support for this idea stems from the fact that the IMM is highly nonlinear and re-estimating the initial condition of the filter based on the current data is effective in terms of accuracy. Simply put, a good re-initialization of the IMM plays a key role for a more accurate calculation of model likelihoods and probabilities, which enables a faster response to model changes and thus reduces peak errors. Furthermore, to maintain a minimum overall computational load, the SE-IMM scheme is applied only during the most critical regimes in which the dominant model changes. This requires incorporation of a suitable model change detection mechanism into the overall decision-estimation scheme. A cumulative sums (CUSUM)-type statistical test is formulated and employed for the problem of model change detection/estimation.

In the history of maneuvering target tracking, hard decision based came to existence first; MM algorithms, which amount to soft decision, took over and have become the vogue of the day, mostly due to the success of IMM algorithm. We believe this paper makes a small step toward explicitly combining the two classes to take advantage of the strength of each class.

The rest of the paper is organized as follows. Section 2 reviews the MM smoothing problem and provides an approximate multiple-step-back IMM smoother. Section 3 outlines the SE-IMM scheme. A CUSUM-type model change detection is formulated in Section 4. Section 5 presents performance evaluation results from the simulation of a maneuvering target tracking scenario. Conclusions are given in Section 6.

\section{MM Smoothing}

Consider the familiar model of hybrid (continuousdiscrete) system in discrete time $k=0,1,2, \ldots$

$$
\begin{aligned}
x_{k+1} & =\Phi_{k+1, k}\left(m_{k+1}\right) x_{k}+w_{k, k+1}\left(m_{k+1}\right) \\
z_{k} & =H_{k}\left(m_{k}\right) x_{k}+v_{k}\left(m_{k}\right)
\end{aligned}
$$


where $x$ is the continuous (base) state with state transition matrix $\Phi, w$ is the random input, $z$ is the measurement, with measurement matrix $H$, and $v$ is the random measurement error. The discrete (modal) state $m_{k} \in \mathbb{M} \triangleq$ $\left\{m^{1}, m^{2}, \ldots, m^{r}\right\}$ is a Markov chain with initial probabilities and transition probability matrix $\Pi=\left(\pi_{i j}\right)_{i, j=1}^{r}$ respectively

$$
\begin{aligned}
P\left\{m_{0}^{j}\right\} & =\mu_{0}^{j} \\
P\left\{m_{k+1}^{j} \mid m_{k}^{i}\right\} & =\pi_{i j} \quad i, j=1, \ldots, r .
\end{aligned}
$$

where $m_{k}^{i} \triangleq\left\{m_{k}=m^{i}\right\}$. The system initial state $x_{0} \sim \mathcal{N}\left[\bar{x}_{0}, P_{0}\right], \quad$ and the white process and measurement noise sequences $\left\{w_{k, k+1}\left(m_{k+1}\right) \sim \mathcal{N}\left[\bar{w}_{k, k+1}, Q_{k, k+1}\left(m_{k+1}\right)\right]\right\}_{k=0,1, \ldots}$ and $\left\{v_{k}\left(m_{k}\right) \sim \mathcal{N}\left[0, R_{k}\left(m_{k}\right)\right]\right\}_{k=0,1, \ldots} \quad$ respectively, are assumed uncorrelated.

It is well known [1] that $\hat{x}_{l \mid k}=E\left[x_{l} \mid z^{k}\right]$ provides the MMSE-optimal estimate of the state $x_{l}$ given measurements $z^{k} \triangleq\left\{z_{0}, z_{1}, \ldots, z_{k}\right\}$. Classically, $\hat{x}_{l \mid k}$ is referred to as predicted, filtered and smoothed estimate iff $l>k, l=k, l<k$ respectively. Theoretically, a formal solution for the smoothing (filtering), (i.e., for $l \leq k$ ) is given by

$$
\hat{x}_{l \mid k}=\sum_{\left\{M^{k}\right\}} \hat{x}_{l \mid k}\left(M^{k}\right) P\left\{M^{k} \mid z^{k}\right\}
$$

where $M^{k} \triangleq\left\{m_{0}, m_{1}, \ldots, m_{k}\right\}$ denotes a modal history and $\hat{x}_{l \mid k}\left(M^{k}\right) \triangleq E\left[x_{l} \mid M^{k}, z^{k}\right]$. For any given modal history $M^{k}$ the optimal smoothed (filtered) estimate $\hat{x}_{l \mid k}\left(M^{k}\right)$ can be obtained by numerous available optimal linear smoothers [17, 16]. $P\left\{M^{k} \mid Z^{k}\right\}$ can also be obtained by means of the Bayes rule, which constitutes formally the optimal solution $[10,1]$. However, this optimal solution has exponentially increasing computation/memory requirements and is thus practically infeasible.

Therefore a number of suboptimal approximations for the MM smoothing problem with polynomial or even linear computational complexity have been proposed $[14,4$, $7,8,5]$. The smoother of [14] is based on pruning more unlikely Markov chain histories - a technique recognized at present as less efficient in comparison with the pseudo-Baysian approximation technique by merging of chain histories. [4] proposed an IMM smoothing by system's time reversion. In [8] two pseudo-Bayesian MM algorithms were developed, which are attractive from computational viewpoint, however they are limited to one-step back smoothing. In [5] a $d$-step back algorithm was proposed, which straightforwardly implements the IMM filtering for an augmented system imbedding the last $d$ system states. This approach is rather involved computationally. In our investigation we established that, for the scenario we simulated, the one-step IMM smoother of [8] and the augmented smoother of [5] provide almost equal accuracy with the former being less computationally demanding than the latter. By this motivation we propose next an extension of the one-step IMM smoother of [8] (referred to as IMMS1) to the case of multiple-step fixed point smoothing, referred to as IMM smoother (IMMS). This implementation is further used, as well as the augmented MM smoother of [5], for the purposes of smoothing-enhanced MM filtering.

The justification of the IMMS is as follows. For the MM smoothing problem at time $l$ given measurement data up to time $k$ the conditional smoothed estimates/covariances and model probabilities are defined with respect to the latest received measurement at time $k$, i.e.,

$$
\begin{aligned}
& \hat{x}_{l \mid k}^{j} \triangleq E\left[x_{l} \mid m_{k}^{j}, z^{k}\right] \quad P_{l \mid k}^{j} \triangleq \operatorname{MSE}\left(\hat{x}_{l} \mid m_{k}^{j}, z^{k}\right) \\
& \mu_{l \mid k}^{j} \triangleq P\left\{m_{l}^{j} \mid z^{k}\right\} \quad j=1, \ldots, r
\end{aligned}
$$

Assume now that for a given $k_{0}$ and $\kappa>k_{0}$ the smoothed (or filtered if $\kappa=k_{0}+1$ ) estimates $\left\{\hat{x}_{k_{0} \mid \kappa-1}^{j}, P_{k_{0} \mid \kappa-1}^{j}, \mu_{k_{0} \mid \kappa-1}^{j}\right\}_{j=1}^{r}$ are available. Then for the time instant $\kappa$ the dynamic system (1) is resumed (reset) at $k_{0}$ as

$$
x_{\kappa}=\Phi_{\kappa, k_{0}}\left(m_{\kappa}\right) x_{k_{0}}+w_{k_{0}, \kappa}\left(m_{\kappa}\right)
$$

with the re-initialization

$$
p\left(x_{k_{0}} \mid z^{\kappa-1}\right) \sim \sum_{j=1}^{r} \mu_{k_{0} \mid \kappa-1}^{j} \mathcal{N}\left[\hat{x}_{k_{0} \mid \kappa-1}^{j}, P_{k_{0} \mid \kappa-1}^{j}\right]
$$

Note that in (8) the state transition matrix $\Phi_{\kappa, k_{0}}\left(m_{\kappa}\right)$ and the process noise $w_{k_{0}, \kappa}\left(m_{\kappa}\right)$ describe the state evolution for the time period starting at $k_{0}$ and ending at $\kappa$ directly (without considering the intermediate states). In other words, it is assumed that there are no model jumps over the interval $\left(k_{0}, k\right]$ (see Section 4$)$.

The Markov transition model becomes

$$
\begin{aligned}
P\left\{m_{k_{0}}^{j} \mid z^{\kappa-1}\right\} & =\mu_{k_{0} \mid \kappa-1}^{j} \\
P\left\{m_{\kappa}^{j} \mid m_{k_{0}}^{i}\right\} & =\pi_{i j}^{\left(\kappa-k_{0}\right)} \quad i, j=1, \ldots, r .
\end{aligned}
$$

where $\pi_{i j}^{\left(\kappa-k_{0}\right)}=\left[\Pi^{\kappa-k_{0}}\right]_{i j}$ are the $\left(\kappa-k_{0}\right)$-step a priori transition probabilities. They can be easily calculated based on the Chapman-Kolmogorov equation via $\Pi^{\left(\kappa-k_{0}\right)}=\Pi^{\kappa-k_{0}}$.

On receipt of a new measurement $z_{\kappa}$ updating of the MM estimates (6)-(7) can be done by the one-step smoother IMMS1 for the reset system (8)-(11) and measurement equation (2) for $k=\kappa$. Thus, an overall IMMS algorithm for calculating at time $k>k_{0}$ the smoothed estimates $\left\{\hat{x}_{k_{0} \mid k}^{j}, P_{k_{0} \mid k}^{j}, \mu_{k_{0} \mid k}^{j}\right\}_{j=1}^{r}$ and $\left\{\hat{x}_{k_{0} \mid k}, P_{k_{0} \mid k}\right\}$ can be obtained, which is given below.

\section{IMMS Algorithm}

For $\kappa=k_{0}+1, \ldots, k$

Step 1. Model-conditional reinitialization 


$$
\begin{aligned}
\hat{x}_{k_{0} \mid \kappa-1}^{0 j} & =\sum_{i=1}^{r} \mu_{\kappa \mid k_{0}}^{i \mid j} \hat{x}_{k_{0} \mid \kappa-1}^{i} \\
P_{k_{0} \mid \kappa-1}^{0 j} & =\sum_{i=1}^{r} \mu_{\kappa \mid k_{0}}^{i \mid j}\left[P_{k_{0} \mid \kappa-1}^{i}+\right. \\
& \left.\left(\hat{x}_{k_{0} \mid \kappa-1}^{i}-\hat{x}_{k_{0} \mid \kappa-1}\right)\left(\hat{x}_{k_{0} \mid \kappa-1}^{i}-\hat{x}_{k_{0} \mid \kappa-1}\right)^{\mathrm{T}}\right] \\
\mu_{\kappa \mid k_{0}}^{i \mid j} & =\frac{1}{\mu_{\kappa \mid k_{0}}^{j}} \pi_{i j}^{\left(\kappa-k_{0}\right)} \mu_{k_{0} \mid \kappa-1}^{i} \\
\mu_{\kappa \mid k_{0}}^{j} & =\sum_{i=1}^{r} \pi_{i j}^{\left(\kappa-k_{0}\right)} \mu_{k_{0} \mid \kappa-1}^{i}
\end{aligned}
$$

Step 2. Model-conditional one-step smoothing

$$
\begin{aligned}
\hat{x}_{k_{0} \mid \kappa}^{j} & =\hat{x}_{k_{0} \mid \kappa-1}^{0 j}+K_{k_{0}, \kappa}^{j} \tilde{z}_{\kappa \mid k_{0}}^{j} \\
P_{k_{0} \mid \kappa}^{j} & =P_{k_{0} \mid \kappa-1}^{0 j}-K_{k_{0}, \kappa}^{j} S_{\kappa \mid k_{0}}^{j} K_{k_{0}, \kappa}^{j \mathrm{~T}} \\
K_{k_{0}, \kappa}^{j} & =P_{k_{0} \mid \kappa-1}^{0 j} \Phi_{\kappa, k_{0}}^{j \mathrm{~T}} H_{\kappa}^{j \mathrm{~T}}\left(S_{\kappa \mid k_{0}}^{j}\right)^{-1}
\end{aligned}
$$

with

$$
\begin{aligned}
& \tilde{z}_{\kappa \mid k_{0}}^{j}=z_{\kappa}-H_{\kappa}^{j} \Phi_{\kappa, k_{0}}^{j} \hat{x}_{k_{0} \mid \kappa-1}^{0 j} \\
& S_{\kappa \mid k_{0}}^{j}=H_{\kappa}^{j} P_{k_{0} \mid \kappa-1}^{0 j} H_{\kappa}^{j \mathrm{~T}}+R_{\kappa}
\end{aligned}
$$

Step 3. Smoothed model probabilities

$$
\begin{aligned}
\mu_{k_{0} \mid \kappa}^{j} & =\frac{1}{\mu_{k_{0} \mid \kappa}} \mu_{k_{0} \mid \kappa-1}^{j} \Lambda_{k_{0} \mid \kappa}^{j} \\
\Lambda_{k_{0} \mid \kappa}^{j} & =\sum_{i=1}^{r} \pi_{j i}^{\left(\kappa-k_{0}\right)} \Lambda_{\kappa \mid \kappa-1}^{i} \\
\mu_{k_{0} \mid \kappa} & =\sum_{j=1}^{r} \mu_{k_{0} \mid \kappa-1}^{j} \Lambda_{k_{0} \mid \kappa}^{j}
\end{aligned}
$$

where $\Lambda_{\kappa \mid \kappa-1}^{i}$ is the likelihood associated with $\tilde{z}_{\kappa \mid \kappa-1}^{j}$ (e.g., given by the direct forward $\operatorname{IMM}[\kappa-1, \kappa]$ ).

Step 4. Smoothed state estimate (output)

$$
\begin{aligned}
& \hat{x}_{k_{0} \mid \kappa}=\sum_{j=1}^{r} \mu_{\kappa \mid \kappa}^{j} \hat{x}_{k_{0} \mid \kappa}^{j} \\
& P_{k_{0} \mid \kappa}=\sum_{j=1}^{r} \mu_{\kappa \mid \kappa}^{j}\left[P_{k_{0} \mid \kappa}^{j}+\right. \\
&\left.\left(\hat{x}_{k_{0} \mid \kappa}^{j}-\hat{x}_{k_{0} \mid \kappa}\right)\left(\hat{x}_{k_{0} \mid \kappa}^{j}-\hat{x}_{k_{0} \mid \kappa}\right)^{\prime}\right]
\end{aligned}
$$

where $\mu_{\kappa \mid \kappa}^{j}$ is the posterior model probability associated with $\tilde{z}_{\kappa \mid \kappa-1}^{j}$ (e.g., given by the direct forward $\operatorname{IMM}[\kappa-1, \kappa])$

Clearly, for $k_{0}=k-1$ the above IMMS algorithm reduces to the IMMS1 algorithm of [8]. More details of the above IMMS algorithm will be given in a forthcoming paper. Here we employ IMMS in the further implementation of smoothing-enhanced IMM filtering discussed next.

\section{Smoothing-Enhanced MM Filter- ing}

A major disadvantage of the smoothing algorithms is the time delay associated with obtaining the smoothed estimates. In most applications however such a delay can not be tolerated and using "future measurements" contradicts with the necessity of "real-time" processing. Naturally in such circumstances arises the idea to use the data gathered up to the current time instant to smooth the estimate for some previous time instant, and then to re-run the estimator from that previous moment with the more accurate smoothed estimate. Clearly, for the optimal linear estimators (e.g., Kalman filter) such an idea is completely senseless, because the current optimal estimate is based on all data and cannot be improved in terms of the same optimality criterion. For the approximate, suboptimal, and highly nonlinear estimators (such as GPB and IMM) this approach however makes good common sense. For example, in a similar manner the smoothing-enhanced re-filtering idea is well used in the so-called iterative EKFs. Different MM estimators differ mainly in the re-initialization of the model conditional filters, which to a large extent determines their performance. For instance, the smarter re-initialization of the IMM scheme leads to a better performance as compared to the GPB1 scheme. In the same spirit, re-initializing an MM estimator with the smoothed estimate from a given previous moment would expectedly provide superior accuracy of the estimate at the current time. In contrast to the pure smoothing, the SE-IMM-estimate is readily available at the current time with additional computation as compared to the filtering.

In this paper we implement and investigate a smoothingenhanced IMM (SE-IMM) scheme, based on an IMM smoother (e.g., the one presented in the previous section, or the IMMS-d algorithm of [5]. More specifically the SE-IMM algorithm at time $k \geq L$ on the receipt of the measurement $z_{k}$ operates as follows.

- Run $I M M S\left[k, k_{0}\right]$ to obtain

$$
\left\{\hat{x}_{k_{0} \mid k}^{j}, P_{k_{0} \mid k}^{j}, \mu_{k_{0} \mid k}^{j}\right\}_{j=1}^{r}
$$

where $k_{0}=k-L$

- For $l=1,2, \ldots, L$

Run $I M M\left[k_{0}+l-1, k_{0}+l\right]$ to obtain

$$
\left\{\hat{x}_{k \mid k}^{j}, P_{k \mid k}^{j}, \mu_{k \mid k}^{j}\right\}_{j=1}^{r}
$$

Experience with the above SE-IMM scheme (see Section 6) indicated that it does considerably reduce the peak errors of the IMM filter during the transitional (model-change) regimes. As a side effect, however, an accuracy degradation during the system steady states (e.g., nonmaneuvering motion) was observed. A possible reason for this could be a larger impact of the erroneous (non-dominant) models to 
the overall estimate due to smoothing. The most natural solution to this issue is to use the above SE-IMM only during the filter transitional regimes. Not only would this provide the best estimates available for both steady state and transitional periods but it also would dramatically decrease the overall computation for the entire estimation interval. Note that as a result of this, we have a combination of soft decision and hard decision. In order to make use of this idea an appropriate model change detection mechanism is indispensable. Estimation of the model change instant is also necessary. This is addressed next.

\section{Model Change Detection}

Information about mode changes is contained in the current model likelihood functions $\Lambda_{k}^{j}=p\left(z_{k} \mid z^{k-1}, x_{k}, m_{k}^{j}\right)$ and the posterior model probabilities $\mu_{k \mid k}^{j}=P\left\{m_{k}^{j} \mid z^{k}\right\}$, both provided by the MM filter. In general, the likelihood, while being more sensitive to model changes, is more prone to random fluctuations and false alarms as compared to the model probabilities. The latter are more conservative and more inert in this regard. Experience about the functioning of MM estimators (e.g., IMM) indicates that during a steady mode of the system (i.e., no jumps occur) typically $^{1}$ one of the MM filter's models is dominant, i.e., it has a probability much larger than the other models. When a jump-wise mode change occurs a jump-wise fall in the likelihood and subsequently in the model probability of the dominant model is observed. If the models are well separated such an MM filter's response can be very informative and very fast at the same time.

One of the simplest possibilities to test for a model change based on the above heuristic considerations is to check for a change in the posterior probability of the dominant mode:

$$
\max _{m^{j} \in \mathbb{M}} \mu_{k \mid k}^{j}<t_{d}
$$

where $t_{d}$ is a dominance threshold (e.g., 0.8), depending on the MM design for the problem under consideration. Once a model change is declared by (21) the reverse test

$$
\max _{m^{j} \in \mathbb{M}} \mu_{k \mid k}^{j} \geq t_{d}
$$

can be used for establishing a steady mode of the filter.

In the context of smoothing-enhanced MM filtering, the tests (21) and (22) could be used to turn the smoothing reinitialization on and off, respectively. Increased robustness and decreased false alarm rate may be achieved by a run test, which requires that (21) (resp. (22)) be satisfied for several (possibly subsequent) times. A better way is to use the ratio of the two highest model probabilities as a test statistic for model dominance [11].

Albeit extremely simple and supported by the common sense, the above test has two major shortcomings. First, it

\footnotetext{
${ }^{1}$ Provided the MM filter is not poorly designed.
}

largely ignores the statistical properties of the time series $\mu_{1 \mid 1}, \mu_{2 \mid 2}, \ldots, \mu_{k \mid k}, \ldots$ Second, it does not give any estimation of the time the change occurred.

Next we formulate a cumulative sum (CUSUM)type statistical test $[9,2,6]$ for model change detection, based on the model probability's time series $\mu_{1 \mid 1}, \mu_{2 \mid 2}, \ldots, \mu_{k \mid k}, \ldots$

The test is run after a dominant model $m^{j_{s}}$ (steady state of the MM filter) is roughly established by (22) at some time $k_{s}$. It considers the hypotheses

$$
\begin{aligned}
& H_{0}=\left\{\text { Model } m^{j_{s}} \text { is in effect for all } k_{s}, k_{s}+1, \ldots, k\right\} \\
& H_{1}=\left\{\text { Model } m^{j_{s}} \text { is not in effect for all } k_{0}, k_{0}+1, \ldots, k\right\}
\end{aligned}
$$

where $k_{0} \in\left(k_{s}, k\right]$ is an unknown instant of change, to be estimated.

Note that there are no jumps over $\left(k_{0}, k\right]$, where $k_{0}$ is the latest moment of model jump. This is consistent with the approximation made in (8) for this application.

Starting from $k_{s}$ the following cumulative sum statistics are computed for $k \geq k_{s}$

$$
\begin{aligned}
\mathcal{S}_{k_{s}} & =0 \\
\mathcal{S}_{k} & =\mathcal{S}_{k-1}+\left(\mu_{k \mid k}^{j_{s}}-\bar{\mu}^{j_{s}}+\lambda \sigma^{j_{s}}\right)
\end{aligned}
$$

where $\bar{\mu}^{j_{s}}$ and $\sigma^{j_{s}}$ are the mean and standard deviation of $\mu_{k \mid k}^{j_{s}}$ if $m^{j_{s}}$ is the dominant mode and $\lambda$ is a design parameter. The decision rule for testing $H_{0}$ vs. $H_{1}$ is

$$
\begin{array}{ll}
\text { Reject } H_{0} & \text { if } \mathcal{S}_{k}-\max _{k_{s} \leq \kappa<k} \mathcal{S}_{\kappa}<-t_{j_{s}} \\
k=k+1 & \text { otherwise }
\end{array}
$$

where $t_{j_{s}}>0$ is a decision threshold. When a model change is detected by (25) the instant of change $k_{0}$ is estimated as

$$
k_{0}=\min \left\{\kappa: \mathcal{S}_{\kappa}=\max _{k_{s}<l \leq k} \mathcal{S}_{l}\right\}
$$

Key design parameters of the above model change detection are the mean $\bar{\mu}^{j}$ and deviation $\sigma^{j}$ of a model $m^{j}$ when it is dominant. In practice they can be evaluated by simulation of steady state (no change) scenarios with true system mode equal to or close to $m^{j}$. The choice of $\lambda$ and the threshold $t$ is discussed in [9].

\section{Simulation: Target Tracking}

\subsection{Tracking Problem}

We simulated the following well-known example, given in complete details in [13]. The target-measurement model is

$$
\begin{aligned}
x_{k+1} & =F x_{k}+G\left[a_{k}+w_{k}\right] \\
z_{k+1} & =H x_{k+1}+v_{k+1}, \quad k=0,1,2, \ldots
\end{aligned}
$$

where $x \triangleq(\mathrm{x}, \dot{\mathrm{x}}, \mathrm{y}, \dot{\mathrm{y}})^{\prime}$ denotes the target state, $a \triangleq(\ddot{\mathrm{x}}, \ddot{\mathrm{y}})^{\prime}$ is the acceleration, $w \sim \mathcal{N}[0, Q]$ is the acceleration process 


\begin{tabular}{|c|c|c|c|c|c|c|c|c|c|}
\hline$k$ & 1 & 31 & 46 & 56 & 81 & 99 & 120 & 140 & 151 \\
\hline \hline$\ddot{x}_{k}$ & 0 & 18 & 2 & 0 & 25 & -2 & 0 & 38 & 0 \\
\hline$\ddot{\mathrm{y}}_{k}$ & 0 & 22 & 37 & 0 & 2 & 19 & -1 & -1 & 0 \\
\hline
\end{tabular}

Table 1: Target Acceleration Scenario

noise, $z=\left(z_{x}, z_{y}\right)^{\prime}$ is the measurement, $v \sim \mathcal{N}[0, R]$ is the random measurement error, and

$$
\begin{aligned}
F & \triangleq\left[\begin{array}{cccc}
1 & T & 0 & 0 \\
0 & 1 & 0 & 0 \\
0 & 0 & 1 & T \\
0 & 0 & 0 & 1
\end{array}\right] \quad G \triangleq\left[\begin{array}{cc}
T^{2} / 2 & 0 \\
T & 0 \\
0 & T^{2} / 2 \\
0 & T
\end{array}\right] \\
H & \triangleq\left[\begin{array}{llll}
1 & 0 & 0 & 0 \\
0 & 0 & 1 & 0
\end{array}\right]
\end{aligned}
$$

The unknown true acceleration $a_{k}=\left(\ddot{x}_{k}, \ddot{y}_{k}\right)^{\prime}$ is assumed piecewise constant with a maximum value of $4 g$ in any direction, i.e.,

$$
\{(\ddot{x}, \ddot{y}):|\ddot{x}|+|\ddot{y}| \leq 40\}
$$

The MM model, used by the estimators, approximates the evolution of the true acceleration via a Markov chain model over a finite set of acceleration values $\left\{a^{1}, a^{2}, \ldots, a^{r}\right\}$ within the assumed range, that is, $P\left\{a_{0}=a^{i}\right\}=P_{i}$ and $P\left\{a_{k}=a^{j} \mid a_{k-1}=a^{i}\right\}=\pi_{i j}$ for $i, j=1,2, \ldots, r$. In our implementations we used the 13-model set design given by

$$
\left\{\begin{array}{lll}
a^{1}=\rho[0,0]^{\prime} & a^{2}=\rho[1,0]^{\prime} & a^{3}=\rho[0,1]^{\prime} \\
a^{4}=\rho[-1,0]^{\prime} & a^{5}=\rho[0,-1]^{\prime} & a^{6}=\rho[1,1]^{\prime} \\
a^{7}=\rho[-1,1]^{\prime} & a^{8}=\rho[-1,-1]^{\prime} & a^{9}=\rho[1,-1]^{\prime} \\
a^{10}=\rho[2,0]^{\prime} & a^{11}=\rho[0,2]^{\prime} & a^{12}=\rho[-2,0]^{\prime} \\
a^{13}=\rho[0,-2]^{\prime} & &
\end{array}\right\}
$$

with $\rho=20 \approx 2 g$, and the same transition probability matrix $\Pi=\left[\pi_{i j}\right]$ as given by (7) of [13]. The other model parameters are $T=1 s, Q=O, R=1250 I, x_{0}=$ $[8000,25,8000,200]^{\prime}$.

The performances of several tracking MM tracking and smoothing algorithms, described further, were investigated over a large number of deterministic maneuver scenarios with fixed acceleration sequences. Deterministic scenarios serve to evaluate algorithms' peak errors, steady-state errors and response times. We present a typical scenario with acceleration values given in Table 1 .

\subsection{Simulation Results}

All simulation results were obtained based on 500 Monte Carlo runs.

Figures 1 through 4 display comparative results of four different smoothers: H1 - one-step back of [8], C1 - onestep back of [5], C2 - two-step back of [5], N2 - the new two-step back, proposed in Section 2. It is seen first that the performances of $\mathrm{H} 1$ and $\mathrm{C} 1$ are practically indistinguishable for this scenario, although these methods are in general based on different underlying approximations. Second, a substantial improvement is seen from the one-step algorithms to the two-step algorithms. This improvement is particularly remarkable regarding the peak errors during model changes, which is of major concern for the MM filters, and empirically supports the idea of smoothing-enhanced MM filtering, investigated in this paper. Third, the performance of the new proposed 2-step back smoothing scheme is almost identical to that of the augmented smoother $\mathrm{C} 2$ [5]. The former however is far less computationally demanding than the latter. From this point of view $\mathrm{N} 2$ seems to be a better choice than $\mathrm{C} 2$.

Figures 5 and 6 present a comparison between the regular IMM and the one-step back smoothing-enhanced IMM (SE-IMM1). Analogous results for the IMM and the two-step back SE-IMM2 are given in Figures 7 and 8. Doubtlessly the SE-IMM gives a superior prediction to the regular IMM during the peak-error periods. This is particularly substantial in the position RMSE plots (Figures 5 and 7). On the other hand, as a side effect of the additional smoothing the error during the steady state (especially non-maneuvering) motion slightly increased in this scenario. Computational considerations and the observation of this phenomenon supported the natural idea to use smoothing-enhanced IMM filtering only during the transitional (model change) regimes of operation. Such processing not only guarantees the best estimates (available from both IMM and SE-IMM) but also dramatically reduces the overall computational load of the algorithm during the entire tracking period. A more or less successful implementation of this idea is demonstrated in Figures 9 and 10. The IMM is compared with an SE-IMM with model-change detection (maneuver on/off logic), which launches SE-IMM only during model changing periods. As seen from the plots the steady state error increasing effect can be mitigated together with a huge computational saving at the same time.

Another observation made from comparing Figures 5 and 6 with Figures 7 and 8, is the small difference between SE-IMM1 and SE-IMM2. It seems that for the tracking scenario considered one-step back smoothing is practically sufficient for the observed peak error reduction.

\section{Conclusion}

An enhanced scheme for IMM estimation by smoothed re-initialization, referred to as smoothing-enhanced IMM (SE-IMM), has been proposed this paper. To facilitate its implementation an approximate IMM smoothing algorithm and an CUSUM-type statistical test for model change de- 


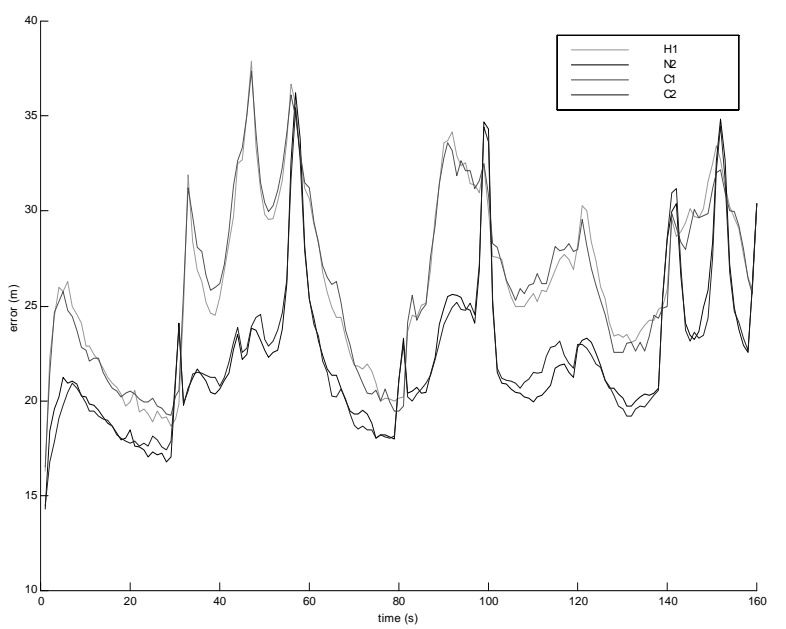

Fig. 1: Smoothing: Position RMSE

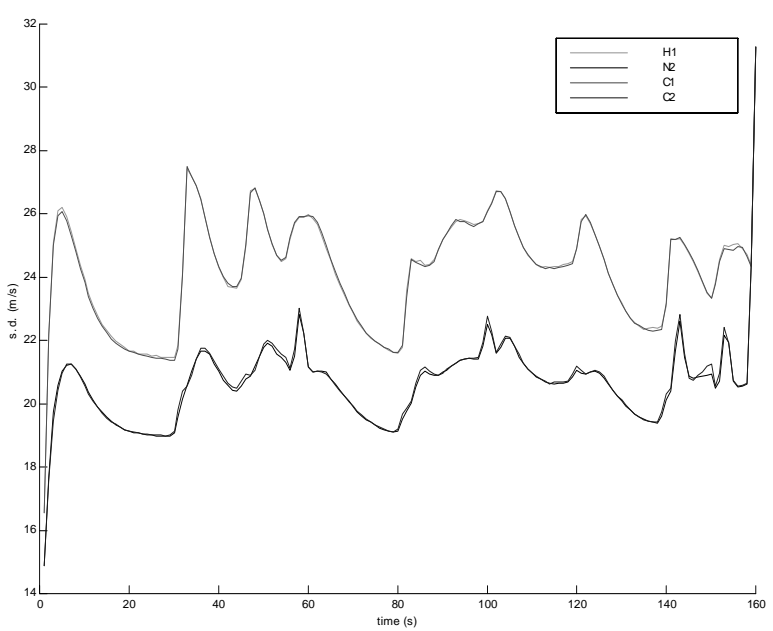

Fig. 2: Smoothing: Position SD

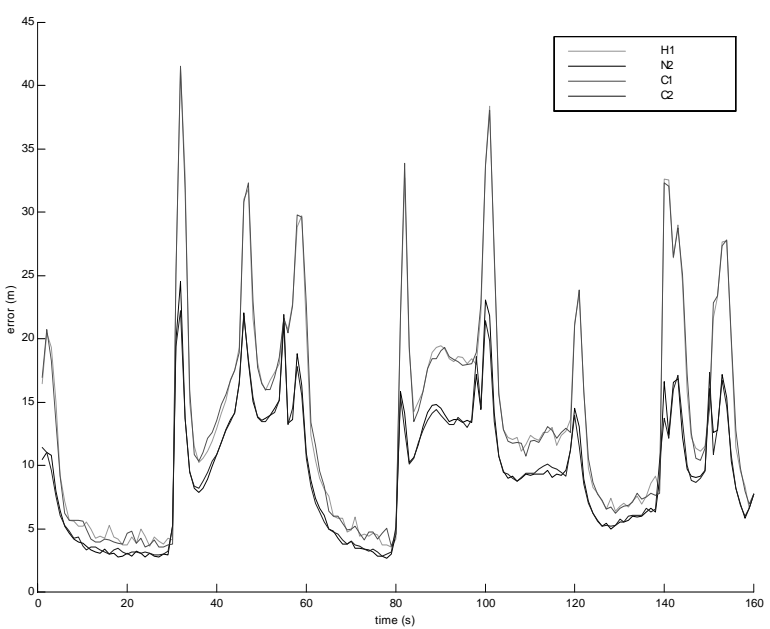

Fig. 3: Smoothing: Velocity RMSE

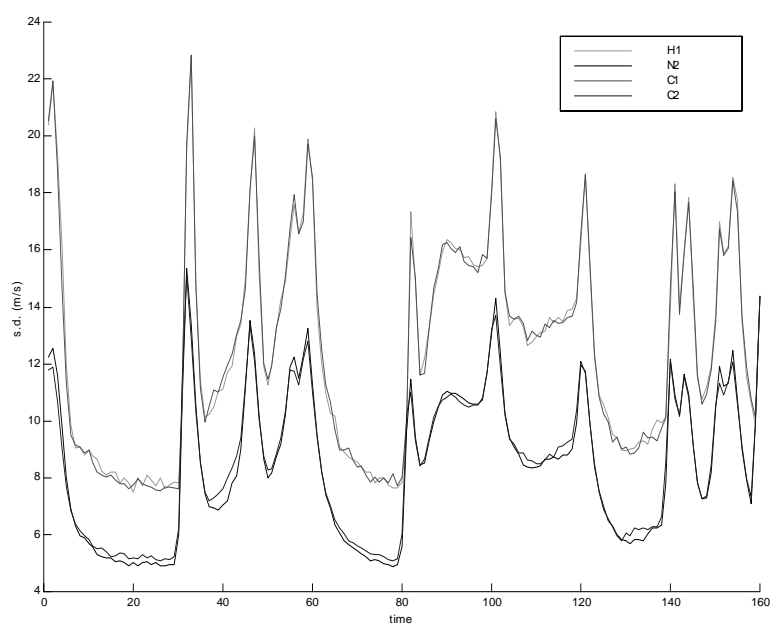

Fig. 4: Smoothing: Velocity SD

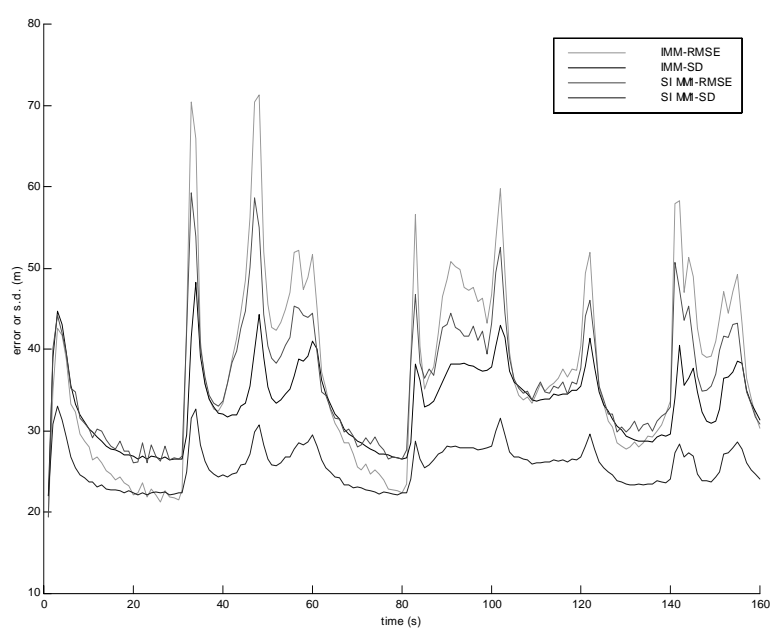

Fig. 5: IMM vs. SE-IMM (1-step back): Position RMSE\&SD

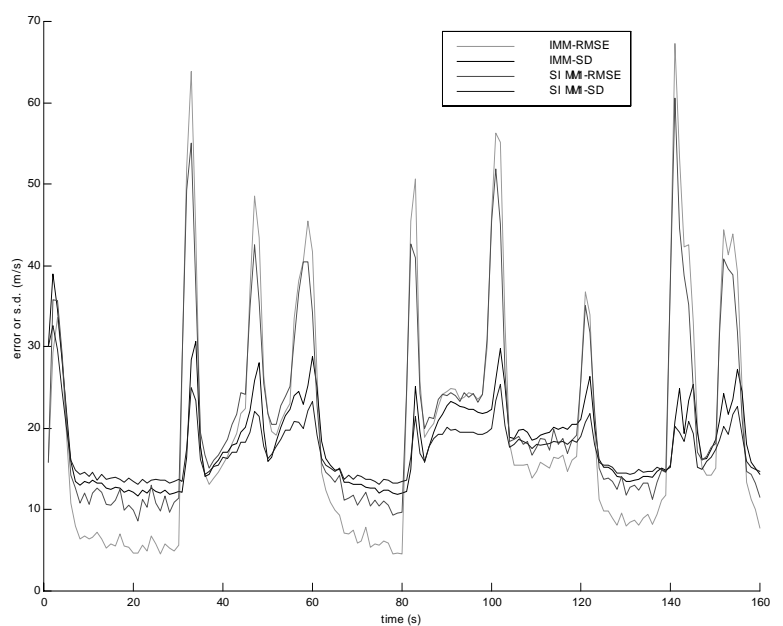

Fig. 6: IMM vs. SE-IMM (1-step back): Velocity RMSE\&SD 


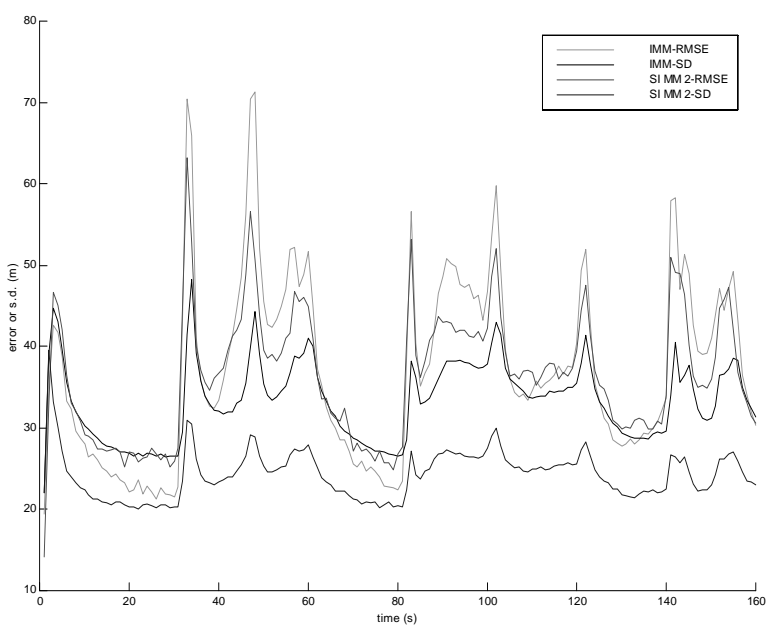

Fig. 7: IMM vs. SE-IMM (2-steps back): Position RMSE\&SD

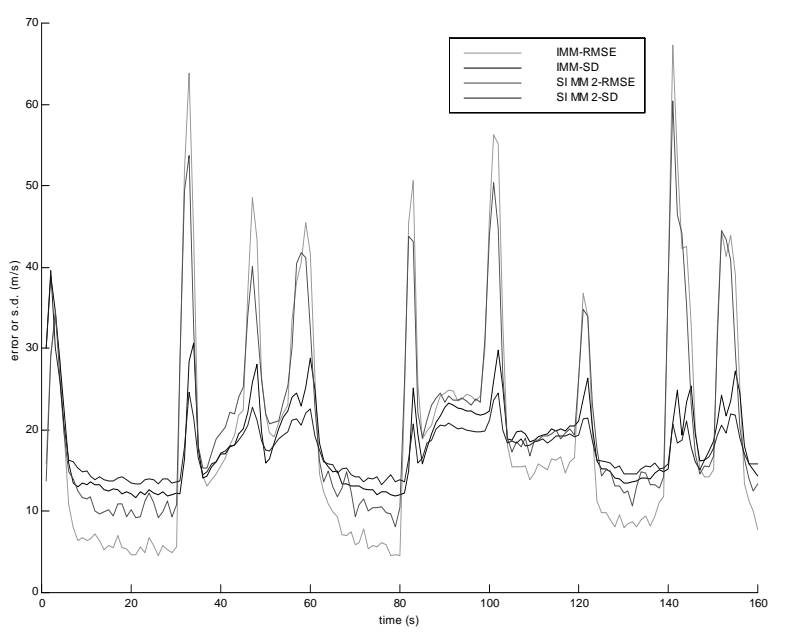

Fig. 8: IMM vs. SE-IMM (2-steps back): Velocity RMSE\&SD

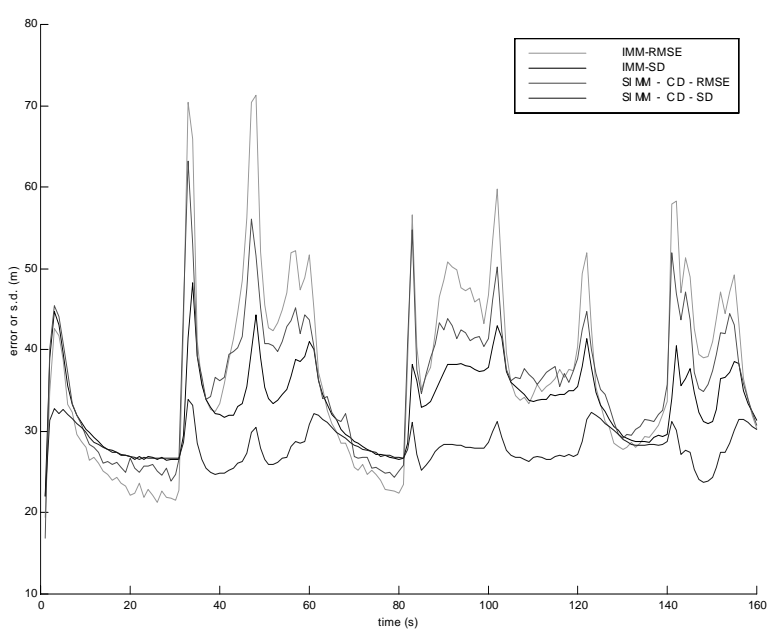

Fig. 9: IMM vs. SE-IMM with CD: Position RMSE\&SD

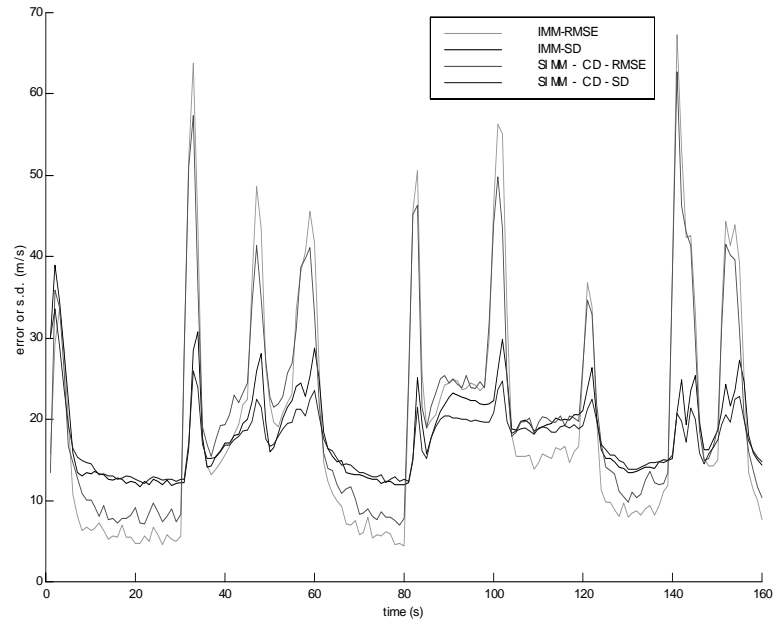

Fig. 10: IMM vs. SE-IMM with CD: Velocity RMSE\&SD

tection have been also provided.

Investigation of the SE-IMM efficiency by simulation has been performed. The simulation results has confirmed that the SE-IMM estimation can be considerably more accurate in comparison with the IMM filter, in transitional (mode-changing) regimes of the dynamic system. At the same time smoothed re-initialization has been observed to yield sometimes accuracy degradation in steady state regimes. Thus, implementation of the SE-IMM with model-change detection, as proposed in the paper, appears a promising solution. An SE-IMM with model-change detection provides the best estimates available from both IMM and SE-IMM which is achieved by a slight increase in computation over the entire estimation period as compared to the standard IMM. In this way, this paper makes the first small step toward combining the soft-decision (MM) and hard-decision based algorithms to take advantage of the strength of each class, as opposed to previously available pure soft-decision (MM) or hard-decision based algorithms [12].

\section{References}

[1] Y. Bar-Shalom, X. R. Li, and T. Kirubarajan, Estimation with Applications to Tracking and Navigation: Theory, Algorithms, and Software. New York: Wiley, 2001.

[2] M. Basseville and I. Nikiforov, Detection of Abrupt Changes: Theory and Application. Englewood Cliffs, NJ: Prentice Hall, 1993.

[3] H. A. P. Blom and Y. Bar-Shalom, "The Interacting Multiple Model Algorithm for Systems with Markovian Switching Coefficients," IEEE Trans. Automatic Control, AC-33(8):780-783, Aug. 1988. 
[4] H. A. P. Blom and Y. Bar-Shalom, "Time-Reversion of a Hybrid State Stochastic Difference System with a Jump-Linear Smoothing Application," IEEE. Trans. Information Theory, IT-36(4):836-847, July 1990.

[5] B. Chen and J. K. Tugnait, "Interacting Multiple Model Fixed-Lag Smoothing Algorithm for Markovian Switching Systems," IEEE Trans. Aerospace and Electronic Systems, 36(1):243-250, Jan. 2000.

[6] F. Gustafsson, Adaptive Filtering and Change Detection. Wiley, 2001.

[7] R. E. Helmick, W. D. Blair, and S. A. Hoffman, "Fixed-Interval Smoothing for Markovian Switching Systems," IEEE. Trans. Information Theory, IT41(6):1845-1855, Nov. 1995.

[8] R. E. Helmick, W. D. Blair, and S. A. Hoffman, "One-Step Fixed-Lag Smoothers for Markovian Switching Systems," IEEE Trans. Automatic Control, AC-41(7):1051-1056, July 1996.

[9] D. V. Hinkley, "Inference about the Change-Point from Cumulative Sum Tests," Biometrika, 58(3):509_ 523, 1971.

[10] X. R. Li, "Hybrid Estimation Techniques," in Control and Dynamic Systems: Advances in Theory and Applications (C. T. Leondes, ed.), vol. 76, pp. 213-287, New York: Academic Press, 1996.

[11] X. R. Li, "Multiple-Model Estimation with Variable Structure-Part II: Model-Set Adaptation," IEEE Trans. Automatic Control, AC-45(11):2047-2060, Nov. 2000.

[12] X. R. Li and V. P. Jilkov, "A Survey of Maneuvering Target Tracking-Part IV: Decision-Based Methods," in Proc. 2002 SPIE Conf. on Signal and Data Processing of Small Targets, vol. 4728, (Orlando, Florida, USA), April 2002.

[13] X. R. Li, Y. M. Zhang, and X. R. Zhi, "MultipleModel Estimation with Variable Structure-Part IV: Design and Evaluation of Model-Group Switching Algorithm," IEEE Trans. Aerospace and Electronic Systems, AES-35(1):242-254, Jan. 1999.

[14] V. J. Mathews and J. K. Tugnait, "Detection and Estimation with Fixed Lag for Abruptly Changing Systems," IEEE Trans. Aerospace and Electronic Systems, AES-19(5):730-739, Sept. 1983.

[15] E. Mazor, A. Averbuch, Y. Bar-Shalom, and J. Dayan, "Interacting Multiple Model Methods in Target Tracking: A Survey," IEEE Trans. Aerospace and Electronic Systems, AES-34(1):103-123, 1996.
[16] J. S. Meditch, "A Survey of Data Smoothing for Linear and Nonlinear Synamic Systems," Automatica, 9(3):151-162, Mar. 1973.

[17] H. E. Rauch, F. Tung, and C. T. Striebel, "Maximum Likelihood Estimation of Linear Dynamic Systems," AIAA Journal, 3:1445-1450, Aug. 1965. 